\title{
Entomofauna associada à couve em pequenas propriedades rurais em Lucas do Rio Verde, Mato Grosso
}

Os insetos associados à couve possuem importância ambiental, uma vez que eles estão ligados ao desenvolvimento direto e indireto desta cultura. A couve está presente na mesa de muitos brasileiros, o que gera demanda ao homem do campo para produzi-la e comercializá-la e são os agricultores familiares os responsáveis por quase toda produção de couve, embora nem sempre estão munidos de conhecimento e assistência técnica. O presente trabalho teve como objetivo identificar a entomofauna associada ao cultivo da couve em 10 pequenas propriedades em Lucas do Rio Verde (Mato Grosso) na área de transição Cerrado-Amazônia. Em cada uma delas, foram realizadas 18 coletas durante maio de 2018 a abril de 2019 , totalizando 180 coletas, realizadas a cada 20 dias. Os insetos foram capturados por meio de armadilhas adesivas amarelas e identificados ao nível de família e, quando possível, identificados ao nível de espécie. Foram coletados 64.194 insetos em 540 armadilhas, pertencentes a 46 famílias, sendo as mais abundantes Ceratopogonidae, Cicadellidae e Aleyrodidae, respectivamente. Foi possível identificar cinco insetos-praga (Agrotis ípsilon, Ascia monuste, Bemisia sp., Diabrotica speciosa e Plutella xylostella), cinco famílias de insetos parasitoides (Braconidae, Chalcididae, Diapriidae, Ichneumonidae e Pteromalidae) e 11 famílias de insetos predadores (Anthocoridae, Carabiidae, Chrysopidae, Coccinelidae, Coreidae, Forficulidae, Mantidae, Pentatomidae, Reduviidae, Syrphidae e Vespidae). Foram identificadas duas guildas, os insetos-predadores e insetos-pragas. A população de insetos associados à couve sofre influência das culturas adjacentes e o conhecimento, monitoramento e conservação dessa diversidade de insetos é importante para a manutenção da cultura no campo, para as tomadas de decisão frente ao controle de pragas bem como possibilita o fortalecimento do uso de práticas conservacionistas e, consequentemente, produtos com maior qualidade.

Palavras-chave: Agricultura familiar; Brassicaceae; Controle; Diversidade; Insecta.

\section{Entomofauna associated with cabbage in small rural properties in Lucas do Rio Verde, Mato Grosso}

\begin{abstract}
The insects associated with the cabbage have environmental importance, since they are linked to the direct and indirect development of this culture. Cabbage is present on the table of many Brazilians, which generates demand for rural people to produce and market it, and family farmers are responsible for almost all cabbage production, although they are not always equipped with knowledge and technical assistance. The present work aimed to identify the entomofauna associated with the cultivation of cabbage in 10 small farms in Lucas do Rio Verde (Mato Grosso) in the Cerrado-Amazon transition area. In each of them, 18 collections were made during May 2018 to April 2019, totaling 180 collections, carried out every 20 days. The insects were captured using yellow adhesive traps and identified at the family level and, when possible, identified at the species level. 64,194 insects were collected in 540 traps, belonging to 46 families, the most abundant of which Ceratopogonidae, Cicadellidae and Aleyrodidae, respectively. It was possible to identify five pest insects (Agrotis ípsilon, Ascia monuste, Bemisia sp., Diabrotica speciosa and Plutella xylostella), five families of parasitoid insects (Braconidae, Chalcididae, Diapriidae, Ichneumonidae and Pteromalidae) and 11 families of predatory insects (Anthocoridae, Carabiidae, Chrysopidae, Coccinelidae, Coreidae, Forficulidae, Mantidae, Pentatomidae, Reduviidae, Syrphidae and Vespidae). Two guilds were identified, insect-predators and insect-pests. The population of insects associated with cabbage is influenced by adjacent crops and the knowledge, monitoring and conservation of this diversity of insects is important for maintaining the culture in the field, for decision making in relation to pest control as well as enabling the strengthening of use conservation practices and, consequently, products with higher quality.
\end{abstract}

Keywords: Family farming; Brassicaceae; Control; Diversity; Insecta.

Topic: Experimentação Agrícola

Reviewed anonymously in the process of blind peer.
Received: 04/10/2020

Approved: $21 / 11 / 2020$
Arthur Yoshio Gemelli (D)

Universidade Federal de Mato Grosso, Brasil

http://lattes.cnpq.br/7881318918301697

http://orcid.org/0000-0002-1387-4247

arthurygemelli@gmail.com

Marliton Rocha Barreto

Universidade Federal de Mato Grosso, Brasil

http://lattes.cnpq.br/4845377498539675

http://orcid.org/0000-0003-3793-8855

mrb.ufmt@gmail.com
Referencing this:

GEMELLI, A. Y.; BARRETO, M. R.. Entomofauna associada à couve em pequenas propriedades rurais em Lucas do Rio Verde, Mato Grosso. Revista Ibero Americana de Ciências Ambientais, v.11, n.6, p.120131, 2020. DOI: http://doi.org/10.6008/CBPC2179$\underline{6858.2020 .006 .0011}$ 


\section{INTRODUÇÃO}

A couve, Brassica oleracea L. (Brassicaceae), é uma cultura muito consumida no país (COSTA et al., 2014) e faz parte da mesa de muitos brasileiros, sendo utilizada para inúmeras receitas e acompanhamentos. Sua produção, no Brasil, está calculada em cerca de 162 mil toneladas por ano (IBGE, 2017), sendo grande parte produzida pela agricultura familiar que é realizada em pequenas propriedades, utilizando mão de obra familiar e com consequente fixação do homem no campo e de comercializada da produção em feiras e mercados, principalmente (LOPES et al., 2018).

Para os agricultores familiares é importante obter em suas lavouras, um ambiente equilibrado, composto por um vasto grupo de seres vivos e, dentro deles os insetos. Os quais desempenham funções diferentes um do outro, seja na atividade de decomposição da matéria orgânica, ciclagem de nutrientes, na polinização, etc., estes insetos compõem o que chamamos de entomofauna (DIEZ-RODRíGUEZ et al., 2017).

O estudo destes insetos e a variação da quantidade destes indivíduos durante o ano nas lavouras, tem grande importância no planejamento da cultura. Ao se identificar os insetos presentes, bem como sua delimitação das épocas de maior aparecimento, é possível o produtor tomar decisões antecipadas, como escolher cultivares a serem utilizadas nos talhões antes da chegada da praga, ou, antecipar aplicações de produtos biológicos e/ou químicos, entre outras tomadas de decisão (DIEZ-RODRíGUEZ et al., 2017). $O$ estudo da entomofauna possibilita ao agricultor compreender a funcionalidade de cada indivíduo presente em seu cultivo, principalmente aqueles que não são pragas, como os insetos benéficos, parasitoides e predadores (DIAS et al., 2017).

As relações ecológicas que ocorrem entre os insetos, plantas e as ações antrópicas, como o manejo integrado da lavoura, influenciam no desenvolvimento da cultura e, principalmente na diversidade da fauna ali presente. Relacionado a isso, surge o termo guildas, proposto por Root (1967), que define como sendo um grupo de espécies, com parentesco taxonômico ou não, que exploram os mesmos recursos naturais, conhecidos também como grupos funcionais.

Para melhor compreender o termo guildas e as relações ecológicas que acontecem entre os insetos, há a necessidade de se estudar a fauna entomológica daquele ambiente. As lavouras de couve, em geral possuem determinados insetos que compõem o seu micro hábitat, como pragas, parasitoides, benéficos, polinizadores e até indicadores de degradação ambiental (DIAS et al., 2017).

A identificação dos insetos presentes em determinados locais tem importância na mensuração dos impactos das ações antrópicas, uma vez que, a qualidade da entomofauna diminui conforme aumenta a atividade humana (AZEVEDO et al., 2011). Os reflexos no desenvolvimento, na qualidade e na quantidade de indivíduos, podem auxiliar na compreensão das melhores alternativas de manejo em locais pouco degradados, como também, recuperar e melhorar a entomofauna de locais degradados, demonstrando a importância em se estudar possíveis grupos ecológicos potenciais.

Devido à importância em levar conhecimento aos agricultores, o presente trabalho teve como objetivo identificar a entomofauna associada ao cultivo da couve em pequenas propriedades na área de 
transição Cerrado-Amazônia.

\section{MATERIAIS E MÉTODOS}

O Trabalho foi realizado em Lucas do Rio Verde, Mato Grosso A região possui clima classificado por Köppen-Geiger como AW, sendo uma estação seca no inverno e uma estação úmida no verão (KÖPPEN et al., 1928). Foram escolhidas 10 propriedades (Tabela 1) que produziam, pelo menos, $2.000 \mathrm{~m}^{2}$ de couve. Em cada propriedade, foram realizadas 18 coletas durante maio de 2018 a abril de 2019, totalizando 180 coletas, realizadas a cada 20 dias. Para cada coleta, foram utilizadas três armadilhas adesivas amarelas $\left(100 \mathrm{~cm}^{2}\right)$ por propriedade. Estas armadilhas ficaram expostas por sete dias na lavoura, e foram distribuídas entre os canteiros da cultura, a uma altura correspondente com a das plantas. Suportes de ferro foram utilizados para fixar as armadilhas, bem como para acompanhar o desenvolvimento (altura) da planta.

Tabela 1: Pontos de coleta (propriedades) para condução do experimento nas lavouras de couve, no município de Lucas do Rio Verde, MT, no período de maio/2018 a abril/2019.

\begin{tabular}{|c|c|c|c|c|c|}
\hline Ponto & Coordenada (S) & Coordenada (O) & Ponto & Coordenada (S) & Coordenada (O) \\
\hline 1 & 12ㅇ'도'15.18' & 555'59.77" & 6 & 130'17.71" & 55ㅇ5'43.66" \\
\hline 2 & $12 \circ 59^{\prime} 10.63^{\prime \prime}$ & 55응' $14.57^{\prime \prime}$ & 7 & $13 \circ 05^{\prime} 30.83^{\prime \prime}$ & $55 \div 53^{\prime} 56,50^{\prime \prime}$ \\
\hline 3 & $12 \div 59^{\prime} 19.67^{\prime \prime}$ & 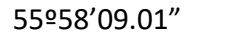 & 8 & 1307'01.84" & $55 \div 54^{\prime} 36.73^{\prime \prime}$ \\
\hline 4 & $13 \circ 04^{\prime} 56.15^{\prime \prime}$ & 555'19.02" & 9 & $13 \circ 07^{\prime} 01.26^{\prime \prime}$ & $5554^{\prime} 51.84^{\prime \prime}$ \\
\hline 5 & $13 \circ 04^{\prime} 27.13^{\prime \prime}$ & 55ㅇ5'ㅇ․ & 10 & $1307^{\prime} 01.06^{\prime \prime}$ & 55ㅇ5'00.19" \\
\hline
\end{tabular}

Depois de retiradas, as armadilhas foram envoltas em filme plástico e levadas ao Laboratório de Entomologia da Universidade Federal de Mato Grosso, Campus Universitário de Sinop, para triagem e identificação dos insetos capturados, o que foi realizado com auxílio de um microscópio estereoscópio. A identificação ocorreu até o nível de família, com auxílio de chaves dicotômicas mais abrangentes, a exemplo de Gallo et al. (2002) e Rafael et al. (2012), além de consulta à bibliografia especializada, para algumas espécies. Os dados coletados foram armazenados em planilha eletrônica e os dados de temperatura e precipitação de Lucas do Rio Verde foram obtidos a partir do site Climate-Data (2019).

\section{RESULTADOS}

Foram coletados 64.194 insetos em 540 armadilhas, sendo identificadas 46 famílias, representando 11 ordens. Tendo como famílias mais representativas Ceratopogonidae (11.713 exemplares), Cicadellidae (9.522 exemplares) e Aleyrodidae (9.232 exemplares) (Tabela 2).

Tabela 2: Ordem, família, números de espécimes e de armadilhas com insetos capturados no período de maio de 2018 a abril de 2019 em cultivos de couve em de Lucas do Rio Verde, MT.

\begin{tabular}{|c|c|c|c|}
\hline Ordem - Subordem & Família & № de espécimes & $\mathrm{N}^{\circ}$ de armadilhas \\
\hline \multirow[t]{2}{*}{ Blattaria } & Kalotermitidae & 6 & 2 \\
\hline & Termitidae & 41 & 17 \\
\hline \multirow[t]{3}{*}{ Coleoptera* } & Carabidae & 206 & 116 \\
\hline & Chrysomelidae & 2.924 & 266 \\
\hline & Coccinelidae & 107 & 79 \\
\hline Dermaptera & Forficulidae & 72 & 33 \\
\hline \multirow[t]{4}{*}{ Diptera* } & Agromyzidae & 18 & 8 \\
\hline & Calliphoridae & 9 & 5 \\
\hline & Ceratopogonidae & 11.793 & 274 \\
\hline & Chloropidae & 29 & 9 \\
\hline
\end{tabular}




\begin{tabular}{|c|c|c|c|}
\hline & Culicidae & 2.062 & 319 \\
\hline & Dolichopodidae & 4.228 & 386 \\
\hline & Drosophilidae & 24 & 3 \\
\hline & Muscidae & 8.089 & 464 \\
\hline & Sarcophagidae & 140 & 48 \\
\hline & Syrphidae & 879 & 145 \\
\hline & Drosophilidae & 725 & 205 \\
\hline Hemiptera Auchernorryncha & Cercopidae & 343 & 113 \\
\hline & Cicadellidae & 9.522 & 410 \\
\hline & Cicadidae & 413 & 143 \\
\hline & Delphacidae & 123 & 24 \\
\hline Hemiptera Heteroptera & Anthocoridae & 415 & 163 \\
\hline & Coreidae & 117 & 53 \\
\hline & Pentatomidae & 15 & 15 \\
\hline & Reduviidae & 7 & 5 \\
\hline Hemiptera Sternorryncha & Aleyrodidae & 9.232 & 142 \\
\hline Hymenoptera* & Apidae & 6 & 5 \\
\hline & Braconidae & 26 & 21 \\
\hline & Chalcididae & 21 & 16 \\
\hline & Diapriidae & 19 & 6 \\
\hline & Formicidae & 1.677 & 264 \\
\hline & Ichneumonidae & 147 & 79 \\
\hline & Pteromalidae & 3 & 3 \\
\hline & Vespidae & 1.462 & 331 \\
\hline Lepidoptera & Noctuidae & 142 & 41 \\
\hline & Nymphalidae & 1 & 1 \\
\hline & Pieridae & 86 & 34 \\
\hline & Plutellidae & 1.256 & 226 \\
\hline & Pyralidae & 71 & 37 \\
\hline & Sphingidae & 4 & 4 \\
\hline Mantodea & Mantidae & 1 & 1 \\
\hline Neuroptera & Chrysopidae & 16 & 5 \\
\hline Orthoptera & Acrididae & 20 & 18 \\
\hline & Gryllidae & 43 & 13 \\
\hline & Tetrigidae & 3 & 1 \\
\hline Thysanoptera & Thripidae & 7.651 & 311 \\
\hline Total & & 64.194 & \\
\hline
\end{tabular}

* = Ordens com famílias ainda não identificadas (6 famílias, 218 insetos).

Com relação a frequência de coleta, os Ceratopogonidae (Diptera: Nematocera), representada principalmente pelo gênero Culicoides sp. Latreille 1809, teve frequência de 50,7\%. Seguidos dos Cicadellidae (Hemiptera: Auchernorryncha), presente em 410 das 540 armadilhas, totalizando $76 \%$ de frequência. Entretanto, foram os representantes dos Muscidae (Diptera) que apresentaram maior frequência (85,9\%), presentes em 464 armadilhas (Figura 1).

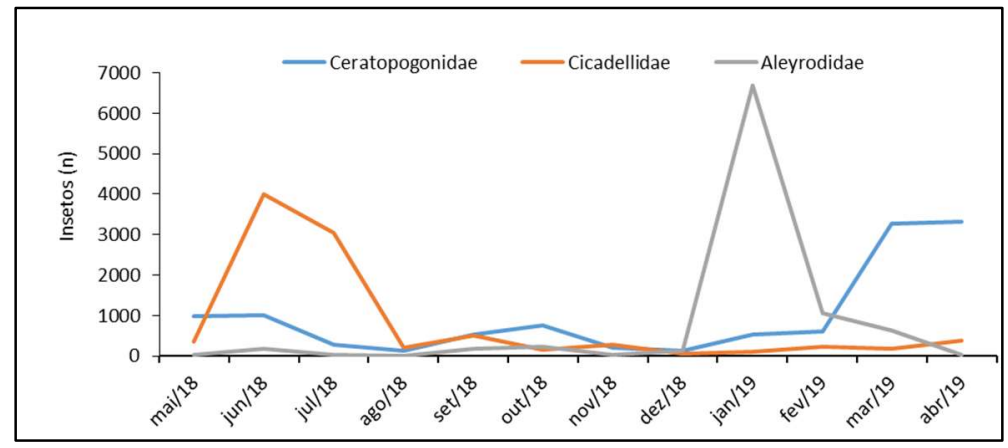

Figura 1: Variação mensal da assembleia de insetos das famílias Ceratopogonidae, Cicadellidae e Aleyrodidae coletados com armadilha adesiva amarela em lavouras de couve no município de Lucas do Rio Verde, no período de maio/2018 a abril/2019.

Referente aos Aleyrodidae (Hemiptera: Sternorryncha), embora tenha sido a terceira família mais 
representativa, e composta principalmente pelo gênero Bemisia spp. Quaintance \& Baker, 1914, seus representantes foram capturados mais abundantemente nos meses de janeiro a março $(6.697,1.069$ e 629 insetos, respectivamente), as demais coletas, tiveram abaixo de 250 indivíduos. Dentre os Chrysomelidae (Coleoptera) capturados, 63\% foram Diabrotica speciosa (Germar, 1824), insetos-praga da cultura da couve. Os demais estão distribuídos entre as famílias Carabidae (206 indivíduos em 116 armadilhas), Coccinelidae (107 indivíduos em 79 armadilhas) e 3 famílias não identificadas (106 indivíduos).

Foram capturadas seis famílias de Lepidoptera (Tabela 2) e a Plutellidae foi o destaque, presente em 42\% das armadilhas, e a traça-das-crucíferas (Plutella xylostella L., 1758) foi a principal espécie capturada (1.186 indivíduos), principalmente na época mais seca, entre os meses de maio a julho. Também, foram coletadas as espécies Ascia monuste orseis Latreille Latreille, 1819 (Pieridae), as Noctuidae Trichoplusia ni Hübner, Agrotis ipsilon Hufnagel, Spodoptera frugiperda Smith, Spodoptera eridania Cramer e Anticarsia gemmatalis Hubner. A família Pyralidae é representada pela Broca-da-couve - Hellula phidilealis Walker.

Referente aos Hemiptera, foram coletados representantes das três sub-ordens: Auchernorryncha, Sternorryncha e Heteroptera, com destaque para as famílias Cicadellidae, cuja maior abundância das cigarrinhas ocorreu nos meses de junho e julho de 2018, que é o período onde as lavouras de milho já estão secas e em fase de colheita; Aleyrodidae, as moscas brancas foram coletadas principalmente entre os meses de janeiro e março de 2019; e Pentatomidae, que de um total de 15 exemplares, três foram exemplares de Podisus nigrispinus Dallas, 1851. Os demais exemplares da família Pentatomidae foram identificados como pragas agrícolas (Euchistos heros Fabricius, Dichelops sp.), de outras culturas como a soja e o milho.

Quanto aos Hymenoptera houve o predomínio de famílias cujos insetos são parasitoides, mais precisamente Braconidae, Chalcididae, Ichneumonidae, Pteromalidae e Diapriidae (Tabela 2). Mas abelhas, formigas e vespas também foram coletadas.

\section{DISCUSSÃO}

Os Culicoides (Ceratopogonidae) são comumente conhecidos como mosquitos-pólvora, maruins ou mosquito-mordedor, estes insetos possuem hábito crepuscular, isto é, são insetos ativos apenas durante o início e final do dia (BAUER et al., 2008). Nos meses de março e abril contatou-se uma elevação do número de indivíduos Ceratopogonidae, possivelmente devido as adubações orgânicas que os produtores fizeram em suas lavouras.

Para isso, Almeida et al. (2017), associa à fauna edáfica, a qual os maruins podem estar presentes (BENTES et al., 2014). No cultivo olerícola a presença dos maruins, possivelmente, está associada ao uso de esterco de animais como vacas e galinhas, que os produtores de couve utilizarem como fertilizante.

Para Trindade et al. (2010), os insetos da família Ceratopogonidae, buscam locais para alimentação rica em proteína animal para o pleno desenvolvimento de seus ovos. Além disso, os maruins desenvolvemse no ciclo da cultura devido à presença de microambientes úmidos nas folhas de couve, onde geralmente há o acúmulo de água de irrigação e/ou chuva, que permite ao mosquito se desenvolver (DIAZ et al., 2019). Esses autores, ainda relataram que o uso da irrigação por aspersão pelos agricultores colabora com a 
formação de microambientes úmidos nas folhas de couve, principalmente na região onde há a união entre folha e caule, a qual cria pequenos orifícios que armazenam água. É neste ambiente em que os insetos ovipositam e dão início a um novo ciclo de desenvolvimento.

Os Agromyzidae são importantes pragas em mais de 25 culturas agrícolas no Brasil e são popularmente conhecidas por moscas minadoras. Os representantes de Chloropidae encontrados são possíveis polinizadores da couve ou de culturas próximas. Quanto aos sirfídeos, Sato (2016) relata que os sirfídeos afidófagos são vorazes e frequentemente ocorrem em grande abundância, sendo que suas larvas podem consumir diversos pulgões em um período de uma a duas semanas. Os Dolichopodidae, outra família de predadores, estão associadas a sistemas de produção de hortaliças, com elevada abundância, quando comparadas com outras famílias de insetos predadores (HARTERREITEN-SOUZA et al., 2014). Os Sarcophagidae presentes estão associados ao esterco utilizado como fertilizante e as demais famílias capturadas acredita-se que estejam associadas em culturas próximas ao cultivo da couve.

A família Cicadellidae, conhecidos popularmente como cigarrinhas, é uma das maiores famílias de insetos da ordem Hemiptera, subordem Sternorryncha, presente no país (RAFAEL et al., 2012), com alguns representantes que atuam como insetos pragas e, ainda, como vetores de doenças viróticas. Apesar do elevado número de exemplares coletados (9.522 indivíduos), os cicadelídeos não são vistos como insetospraga na cultura da couve no Brasil (CELESTINO et al., 2015; BRASIL, 2019). Mas estudos de Eckstein et al. (2014) e Kreycy et al. (2018) tem associado algumas espécies de cicadelídeos como vetores de viroses em couve.

O número de cigarrinhas capturados durante o ciclo da couve pode estar associada ao cultivo do milho, onde as culturas estão próximas ou até mesmo dentro da mesma propriedade, uma vez que a região de estudo é grande produtora de cereais como milho (Zea mays L.) e soja (Glycine max L. Merril) (DALLACORT et al., 2019). Segundo relatos de Hill et al. (2019) a família Cicadellidae inclui pragas em potencial para a cultura do milho como a Dalbulus maidis DeLong \& Wolcott e a Deois flavopicta Stal.

Neste trabalho a maior abundância das cigarrinhas ocorreu no período onde as lavouras de milho já estão secas e em fase de colheita. É provável, que o aumento da população se deve ao fato da ocorrência da colheita e senescência das lavouras de milho, o que obriga as cigarrinhas a procurarem outros locais de refúgio (ÁVILA et al., 2008). Além de procurarem outros abrigos, nesse período, as cigarrinhas estão sob forte pressão pela procura por alimento. Na época seca na região ocorrem os menores valores de precipitação anual (CLIMATE-DATA, 2019), resultando em uma região com poucas culturas verdes, sobrando como alimento e/ou refúgio, apenas as culturas irrigadas como a couve, alface, salsinha, entre outras.

Quanto aos Aleyrodidae (Hemiptera: Sternorryncha), a espécie Bemisia tabaci Gennadius 1889, popularmente conhecida como mosca branca, é a terceira família com maior número de exemplares. As moscas brancas são insetos fitófagos, que se alimentam de plantas, no caso da couve, as moscas brancas são pragas para a cultura (BRASIL, 2019).

O principal período de coleta de moscas brancas ocorreu entre janeiro e março de 2019. Estes surtos de mosca branca na região de estudo ocorrem devido ao plantio em larga escala da soja. Entre os meses de 
setembro e fevereiro, ocorre o plantio da soja no município e, a mosca branca costuma aparecer em meados de janeiro e fevereiro, quando ocorre o florescimento e enchimento de grãos de soja. Em trabalho realizado por Melo et al. (2015), no município de Lucas do Rio Verde, a mosca branca trouxe perdas de até $60 \%$ na região, aparecendo durante o cultivo da soja.

A Diabrotica speciosa, popularmente conhecida como vaquinha ou larva-alfinete, é praga na cultura da couve (PRATISSOLI, 2019). Para Milleo et al. (2013) a D. speciosa possui variação temporal ano a ano, o que pode indicar que em outros anos, variações como está podem ocorrer em meses distintos do encontrado aqui. Além disso, para os mesmos autores, os fatores climáticos (precipitação, umidade relativa e temperatura) não foram capazes de se correlacionar com a variação populacional da praga.

Sato (2016) cita que os Coleoptera Coccinellidae e Carabidae como predadores de maior ocorrência em couve. As famílias de coleópteros coletados apresentam insetos predadores, que se alimentam de uma diversidade de insetos como os lepidópteros das famílias Plutellidae e Noctuidade (SUENAGA et al., 2001), e outros invertebrados. Já a família Coccinelidae, popularmente conhecido como "joaninhas", é formada por insetos entomófagos, alimentam-se de larvas de outros insetos, como besouros, ácaros e pulgões (HOLTZ et al., 2015) e as joaninhas estão presentes na cultura da couve fazendo a predação de ácaros e pulgões. Para Resende et al. (2009) consórcios de couve com coentro favorece a população e a diversidade de espécies de joaninhas, uma vez que o coentro fornece recursos alimentares (pólen), locais de oviposição, proteção para ovos, pupas e adultos. Em todas as 10 propriedades avaliadas, havia o cultivo de coentro, embora não em consórcio, a presença do coentro pode afetar positivamente a presença das joaninhas no cultivo da couve.

Em lepidópteros a fase larval é responsável por atacar as folhas de couve (BORTOLI et al., 2014). A família Plutellidae, representada principalmente pela traça-das-crucíferas (Plutella xylostella), com 1.186 exemplares coletados, possui relatos no Brasil desde 1928 (MEDEIROS et al., 2003) e está distribuída por quase todo o mundo, causando prejuízos nas lavouras e comprometendo toda a produção (SYED et al., 2018). As traças, capturadas sobretudo na época mais seca do ano corrobora com Marchioro et al. (2011) e Celestino et al. (2015), onde é nos períodos mais secos do ano a maior ocorrência da traça-das-crucíferas no campo.

Com relação as demais lepidópteros pragas, Ascia monuste - comumente conhecida como curuquerê-da-couve - causa severos prejuízos nas lavouras (HOLTZ et al., 2015). Assim como os representantes das famílias Noctuidae (LABBÉ et al., 2011; SOUZA et al., 2015; RODRIGUES et al., 2015; MICHEREFF-FILHO et al., 2008; FRAGOSO et al., 2015) e Pyralidae, aqui representada pela Hellula phidilealis (Broca-da-couve), que diferentemente das demais famílias de Lepidoptera, ela ataca o meristema apical da couve, provocando o aparecimento de brotações e posterior secamento da planta (RODRIGUES et al., 2015).

Alguns percevejos da família Pentatomidae foram coletados, sendo esta família responsável por várias pragas de culturas agrícolas, como por exemplo a soja e o milho (SILVA et al., 2014). Para a couve, os pentatomídeos não possuem representante de inseto-praga, mas sim de insetos predadores como o $P$. nigrispinus, que podem atuar como predador natural de pragas das crucíferas, e exemplo da traça-dascrucíferas (TORRES et al., 2010; DIBELLI et al., 2013; GRIGOLLI et al., 2017). Referente aos Anthocoridae e Coreidae capturados, seus representantes estão associados à predação, tanto da traça-das-crucíferas em 
diversas brassicaceas (THULER et al., 2007) como de outras culturas adjacentes a couve.

A traça-das-crucíferas possui diversos inimigos naturais, principalmente parasitoides da ordem Hymenoptera, mais precisamente das famílias Braconidae (BERTOLACCINI et al., 2011; CHAU et al., 2019), Chalcididae (TORRES et al., 2010; BERTOLACCINI et al., 2011), Ichneumonidae (POURIAN et al., 2015; GAVILANEZ et al., 2019), sendo todas estas famílias capturadas nas armadilhas, além de Pteromalidae e Diapriidae. As famílias Ichneumonidae e Braconidae são as famílias comumente encontradas nas lavouras, atuando no parasitismo da $P$. xylostella, além disso, são as famílias de maior efetividade no controle da traça (CELESTINO et al., 2015). Em função da presença de parasitoides nas lavouras, os agricultores poderiam ser estimulados a darem condições para a manutenção destes indivíduos, e assim diminuir o uso de agrotóxicos e os impactos ambientais atrelado aos agrotóxicos (GUIMARÃES et al., 2012). Quanto aos Apidae e Vespidae coletados, os mesmos foram apontados como possível polinizador, a exemplo do que ocorrem com exemplares dessas famílias em Canola (Brassica napus) (Cruciferae) conforme Mussury et al. (2003).

Possivelmente, a riqueza de espécies encontrada na couve esteja associada com a vegetação natural bem como as demais culturas presentes na área de estudo, as quais promovem disponibilidade de alimento, abrigo e reprodução, com consequente favorecimento para os insetos presentes, potencializado a oviposição (VAN RIJN et al., 2013).

Os insetos capturados neste trabalho podem ser divididos em guildas, sendo os principais a de insetos predadores e a de insetos pragas. Baseado na amplitude do termo guildas, compreender as estruturas tróficas ali presentes no cultivo possibilita aos agricultores delimitar quais recursos alimentares estão sendo explorados pelos insetos. Além disso, possibilita também o fortalecimento do uso de práticas conservacionistas e consequentemente produtos com maior qualidade.

A guilda de insetos pragas da couve corresponde por 10.716 insetos, sendo formada pelas famílias Plutellidae (1.256), Pieridae (86), Chrysomelidae (2924), Aleyrodidae (9.232) e Noctuidae (142), com uma representatividade de $16,6 \%$ dos insetos capturados. É interessante frisar que as cinco famílias que contém insetos pragas da couve, também possuem espécies pragas em outras culturas como alface, rúcula, milho. Portanto sua presença pode estar ligada ao fato de outras culturas estarem presentes na propriedade. Diferente delas, a P. xylostella está ligada diretamente à presença de culturas brássicas. Mas para Holtz et al. (2015) a traça sobrevive em outras culturas, mas não chega a afetar o desenvolvimento destas.

A segunda guilda de insetos com maior representatividade é a dos predadores, composta por 3.297 insetos, distribuídos em 11 famílias, sendo elas: Carabiidae (206), Coccinelidae (107), Forficulidae (72), Syrphidae (879), Anthocoridae (415), Coreidae (117), Pentatomidae (15), Reduviidae (7), Vespidae (1.462), Chrysopidae (16) e Mantidae (1), com uma representatividade de 5,30\%.

Representantes de Carabiidae e Pentatomidae podem predar larvas de $P$. xylostella (SUENAGA et al., 2001; GRIGOLLI et al., 2017). Os insetos desta guilda possuem uma ligação direta com o manejo integrado de pragas das culturas, sendo responsáveis por realizar o controle biológico (CARDOSO et al., 2010).

Para a guilda dos predadores, os recursos disponíveis são as próprias pragas e demais insetos presentes na cultura da couve, ao passo de que, a manutenção destas famílias durante todo o 
desenvolvimento da cultura deve auxiliar o agricultor no controle e no manejo das diversas pragas presentes. A introdução de culturas como coentro, endro e funcho, podem trazer aumento desta guilda, uma vez que estas culturas apresentam aumento na quantidade de insetos predadores, devido a disponibilização de recursos alimentares (RESENDE et al., 2012).

Se comparado em números, a quantidade de insetos predadores é menor que a quantidade de insetos pragas, porém, a diversidade no número de famílias de predadores é $240 \%$ maior que pragas, refletindo em maior e melhor chance que ocorra controle de pragas. Essa diversidade de insetos predadores pode estar relacionada com interações entre as diversas culturas adjacentes as lavouras de couve neste trabalho, culturas como tomate, alface, abobrinha, pepino, milho, coentro e crotalária, eram vistos em grande parte das propriedades estudadas.

A riqueza da entomofauna, desde que conhecida pelos agricultores, pode auxiliá-lo no controle natural, no manejo integrado de pragas, minimizando o uso de inseticidas e diminuindo os riscos para a saúde de quem produz e de quem consome. As diversas famílias de insetos encontradas mostram a necessidade do uso de práticas conservacionistas para que se possam potencializar seus efeitos. Uma vez que o uso inadequado de agrotóxicos nas lavouras pode estar diminuindo a diversidade de insetos presentes, causando desequilíbrio na fauna (FIGUEIREDO, 2014).

\section{CONCLUSÕES}

Dos insetos presentes na cultura da couve, capturados com armadilha adesiva, 16,6\% correspondem as pragas e $5,30 \%$ aos insetos predadores. Porém a diversidade no número de famílias de predadores foi $240 \%$ maior que a de pragas. Os insetos envolvidos na cultura - parasitoides e predadores - se bem manejados podem auxiliar o produtor a produzir alimentos de melhor qualidade, principalmente com menor quantidade de agrotóxicos. É importante levar até os pequenos produtores conhecimentos técnicos e fundamentos teóricos para melhorar a preservação e a manutenção da entomofauna.

\section{REFERÊNCIAS}

ALMEIDA, H. S.; SILVA, R. F.; GROLLI, A. L.; SHEID, D. L.. Ocorrência e diversidade da fauna edáfica sob diferentes sistemas de uso do solo. Revista Brasileira de Tecnologia Agropecuária, Frederico Westphalen, v.1, n.1, p.15-23, 2017.

ÁVILA, C. J.; ARCE, C. C. M.. Flutuação populacional da cigarrinha-do-milho em duas localidades do Mato Grosso do Sul. Ciência Rural, Santa Maria, v.38, n.4, p.1129-1132, 2008. DOI: http://dx.doi.org/10.1590/S0103-84782008000400035

AZEVEDO, F. R.; MOURA, M. A. R.; ARRAIS, M. S. B.; NERE, D. R.. Composição da entomofauna da Floresta Nacional do Araripe em diferentes vegetações e estações do ano. Revista Ceres, Viçosa, v.58, n.6, p.740-748, 2011.

BAUER, M. L. F.; CÁCERES, A.; SILVA, C. S.; BAZAN, W. V.; PERES, A. G.; COSTA, J. M.. New records of Culicoides Latreille (Diptera: Ceratopogonidae) from Peruvian Amazonian region. Biota Neotropica, Campinas, v.8, n.2, p.33-38, 2008. DOI: https://doi.org/10.1590/S1676-

\section{$\underline{06032008000200002}$}

BENTES, S. P. C.; HAMADA, N.; KEPPLER, R. L. F. Caracterização morfológica de ovos de insetos aquáticos e seus habitats na Amazonia Central, Brasil. In: HAMADA, N.; NESSIMIAN, J. L.; QUERINO, R. B.. Insetos aquáticos na Amazônia brasileira: Taxonomia, biologia e ecologia. Manaus: INPA, 2014.

BERTOLACCINI, I.; SÁNCHES D. E.; ARREGUI, M. C.; FAVARO, J. C.; THEILER, N.. Mortality of Plutella xylostella (Lepidoptera, Plutellidae) by parasitoids in the Province of Santa Fe, Argentina. Revista Brasileira de Entomologia, v.55, n.3, p.454-456, 2011. DOI: http://dx.doi.org/10.1590/S0085$\underline{56262011005000029}$

BORTOLI, S. A.; CARVALHO, J. S.; VACARI, A. M.; GOULART, R. M.. Consumo foliar da traça-das-crucíferas em couve e brócolis tratados com sinigrina. Arquivos do Instituto Biológico, São Paulo, v.81, n.3, p.264-271, 2014. DOI: 
http://dx.doi.org/10.1590/1808-1657000672012

BRASIL. Agrofit: Sistema de Agrotóxicos Fitossánitários do Ministério da Agricultura, Pecuária e Abastecimento. MAPA, 2019.

CARDOSO, M. O.; PAMPLONA, A. M. S. R.; MICHEREFFFILHO, M.. Recomendações técnicas para o controle de lepidópteros-praga em couve e repolho no Amazonas. Circular técnica 35. Manaus: Embrapa Amazônia Ocidental, 2010.

CELESTINO, F. N.; SOUZA, L. P.; VALBON, W. R.; BESTETE, L. R.; RONDELLI, V. M.. Traça-das-crucíferas (Plutella xylostella). In: HOLTZ, A. M.; RONDELLI, V. M.; CELESTINO, F. N.; BESTETE, L. R.; CARVALHO, J. R.. Praga das Brássicas. Colatina: IFES, 2015.

CHAU, N. N. B.; KIEU, N. T. P.; DUNG, N. V. T.; QUOC, N. B.; PHUONG, T. K. Effects of floral resources on the longevity and parasitism of Cotesia vestalis Haliday (Hymenoptera: Braconidae) on Plutella xylostella (L.) (Lepidoptera: Plutellidae) in Vietnam. Heliyon, v.5, n.8. 2019. DOI: https://doi.org/10.1016/j.heliyon.2019.e02258

CLIMATE-DATA. Clima de Mato Grosso. Lucas do Rio Verde. Gráfico de temperatura e precipitação anual de Mato Grosso. Clima-data, 2019.

COSTA, E. M. R.; MARCHESE, A.; MALUF, W. R.; SILVA, A. A.. Resistance of kale genotypes to the green peach aphid and its relation to leaf wax. Revista Ciência Agronômica, Fortaleza, v.45, n.1, p.146-154, 2014. DOI: http://dx.doi.org/10.1590/S1806-66902014000100018

DALLACORT, R.; BARBIERI, J. D.; TIEPPO, R. C.. Impacts of Future Climate Predictions on Second Season Maize in an Agrosystem on a Biome Transition Region in Mato Grosso State. Revista Brasileira de Meteorologia, v.34, n.2, p.335347, 2019. DOI: http://dx.doi.org/10.1590/010277863340241

DIAS, C.B.R.; GERVÁSIO, R.C.R.G.; FREITAS, H. R.; BARROSO, K. A.. Levantamento de hortas urbanas e registro da entomofauna associada a esses ambientes no município de Petrolina - PE. EXTRAMUROS - Revista de Extensão da UNIVASF. Petrolina, v.5, n.2, p.114-124, 2017.

DIAZ, F.; MANGUDO, C.; GLEISER, R. M.; MONDEIROS, M. M.. Re-descrição de imaturos de Dasyhelea flavifrons GuérinMéneville (Culicomorpha: Ceratopogonidae) e nova contribuição para o conhecimento de seus habitats larvais. Anais da Academia Brasileira de Ciências, Rio de Janeiro, v.91 n.1, 2019. DOI: http://dx.doi.org/10.1590/0001$\underline{3765201920180047}$

DIBELLI, W.; BORTOLI, S. A.; VOLPE, H. X. L.; VACARI, A. M.; MAGALHÃES, G. O.; DUARTE, R. T.; POLANCZYK, R. A.. Effect of Bacillus thuringiensis on the Biological Parameters and Phytophagy of Podisus nigrispinus (Hemiptera: Pentatomidae). Entomologia Generalis, v.34, n.4, p.313-321. DOI: http://doi.org/10.1127/entom.gen/34/2013/313

DIEZ-RODRÍGUEZ, G. I.; SOSINSKI, E. E.; HÜBNER, L. K.; ANTUNES, L. E. C.; NAVA, D. E.. Entomofauna associated to different phenological stages on blueberry crop. Revista Brasileira de Fruticultura, v.39, n.5, e-384, 2017. DOI: http://dx.doi.org/10.1590/0100-29452017384

ECKSTEIN, B.; BARBOSA, J. C.; KREYCI, P. F.; ZANOL, K. M. R.; COELHO, L. B. N.; GONÇALVES, A. C. S. M. L.; BRUNELLI, K. R.; LOPES, J. R. S.; BEDENDO, I. P.. Identification of potential leafhoppers vectors of phytoplasmas (16Srlll group) associated with broccoli stunt disease in Brazil. Australasian Plant Pathology, v.43, p.459-463, 2014. DOI: https://doi.org/10.1007/s13313-014-0293-8

FIGUEIREDO, M.. Guerra no campo: Agrotóxicos são aliados ou ameaça?. Revista Ciência e Cultura, São Paulo, v.66, n.2, 2014. DOI: http://dx.doi.org/10.21800/S00096725201400020000

FRAGOSO, D. F. M.; CARVALHO, J. R.; BARROS, A. P.; COFLER, T.; MARCHIORI, J. J. P.. Lagarta-das-folhas (Spodoptera eridania). In: HOLTZ, A. M.; RONDELLI, V. M.; CELESTINO, F. N.; BESTETE, L. R.; CARVALHO, J. R.. Praga das Brássicas. Colatina: IFES, 2015

GALLO, D.; NAKANO, O.; SILVEIRA NETO, S.; CARVALHO, R. P. L.; BATISTA, G. C.; BERTI FILHO, E.; PARRA, J. R. P.; ZUCCHI, R. A.; ALVES, S. B.; VENDRAMIN, J. D.; MARCHINI, L. C.; LOPES, J. R. S.; OMOTO, C.. Entomologia agrícola. Piracicaba: FEALQ, 2002. p.920

GAVILANEZ, R. E.; GRILLI, M. P.. First record of the spatiotemporal variation of Plutella xylostella (Lepidoptera: Plutellidae) and its parasitoids complex in the horticultural area of Córdoba city in central Argentina. Biological Control, v.133, p.1-8, 2019. DOI: https://doi.org/10.1016/j.biocontrol.2019.02.017

GRIGOLLI, J. F. J.; GRIGOLLI, M. M. K.; RAMALHOC, D. G.; MARTINS, A. L.; VACARI, A. M.; BORTOLI, S. A.. Phytophagy of the predator Podisus nigrispinus (Dallas, 1851) (Hemiptera: Pentatomidae) fed on prey and Brassicaceae. Brazilian Journal of Biology, v.77, n.4, p.703-709, 2017. DOI: http://dx.doi.org/10.1590/1519-6984.16615

GUIMARÃES, J. A.; ABREU, D. V.; VIDAL, M. C.; MOURA, A. P.; MICHEREFF-FILHO, M.. Entomofauna associada a quatro espécies de plantas aromáticas. Horticultura Brasileira, v.30, n.2, p.6420-6425, 2012.

HARTERREITEN-SOUZA, É. S.; TOGNI, P. H. B.; PIRES, C. S. S.; SUJII, E. R.. The role of integrating agroforestry and vegetable planting in structuring communities of herbivorous insects and their natural enemies in the Neotropical region. Agroforest Systems, v.88, n.2, p.205219, 2014. DOI: http://doi.org/10.1007/s10457-013-9666-1

HILL, J. G.; ALBARRACIN, E. L.; ARAOZ, M. V. C.; VIRLA, E. G. Effects of host species and host age on biological parameters of Anagrus virlai T (Hymenoptera: Mymaridae), an egg parasitoid of Dalbulus maidis (Hemiptera: Cicadellidae) and Peregrinus maidis (Hemiptera: Delphacidae). Biological Control, v.131, p.74-80, 2019. DOI: https://doi.org/10.1016/j.biocontrol.2018.12.002

HOLTZ, A. M.; RONDELLI, V. M.; CELESTINO, F. N.; BESTETE, L. R.; CARVALHO, J. R.. Praga das Brássicas. Colatina: IFES, 2015.

IBGE. Instituto Brasileiro se Geografia e Estatística. Censo Agropecuário de 2017. Rio de Janeiro: IBGE, 2017. 
KÖPPEN, W.; GEIGER, R.. Klimate der Erde. Gotha: Verlag Justus Perthes. 1928.

KREYCY, P. F.; ECKSTEIN, B.; LOPES, J. R. S.; FERREIRA, J.; BEDENDO, I. P.. Transmission of "Candidatus Phytoplasma pruni" related strain associated with broccoli stunt by four species of leafhoppers. Journal of Phytopathology, v.166, p.502-505. DOI: https://doi.org/10.1111/jph.12710

LABBÉ, R.; CAVENEY, S.; DONLY, C.. Expression of multidrug resistance proteins is localized principally to the Malpighian tubules in larvae of the cabbage looper moth, Trichoplusia ni. The Journal of Experimental Biology, v.214, n.6, p.937944, 2011. DOI: https://doi.org/10.1242/jeb.051060

LOPES, C. V. A.; ALBUQUERQUE, G. S. C.. Agrotóxicos e seus impactos na saúde humana e ambiental: uma revisão sistemática. Saúde em Debate, Rio de Janeiro, v.42, n.117, p.518-534. 2018. DOI: http://dx.doi.org/10.1590/0103$\underline{1104201811714}$

MARCHIORO, C. A.; FOERSTER, L. A.. Development and Survival of the Diamondback Moth, Plutella xylostella (L.) (Lepidoptera: Yponomeutidae) as a Function of Temperature: Effect on the Number of Generations in Tropical and Subtropical Regions. Neotropical Entomology, v.40, n.5, p.533-541, 2011. DOI:

http://dx.doi.org/10.1590/S1519-566X2011000500003

MEDEIROS, P. T.; DIAS, J. M. C. S.; MONNERAT, R. G.; SOUZA, N. R.. Instalação e manutenção de criação massal da Traçadas-crucíferas (Plutella xylostella). Circular Técnica 29. Brasília: Embrapa Recursos Genéticos e Biotecnologia, 2003.

MELO, W. C.; YAMASHITA, O. M.; CARVALHO, M. A. C.; DALLACORT, R.; TEIXEIRA, S. O.. Produtividade de soja em função de diferentes épocas de dessecação no município de Lucas do Rio Verde-MT. Enciclopédia Biosfera, Goiânia, v.11, n.21, p.1564-1575, 2015.

MICHEREFF-FILHO, M.; TORRES, J. B.; ANDRADE, L. N. T.; NUNES, M. U. C.. Effect of some biorational insecticides on Spodoptera eridania in organic cabbage. Pest Management Science, v.64, p.761-767, 2008. DOI:

https://doi.org/10.1002/ps.1554

MILLEO, J.; SOUZA, J. M. T.; BARBOLA, I. F.; MOURA, L. A.; PUCCI, M. B.. Diversidade e sazonalidade de crisomelídeos (Coleoptera: Chrysomelidae) em pomar, no município de Ponta Grossa, Paraná, Brasil. Revista Brasileira de Fruticultura, Jaboticabal, v.35, n.2, 2013. DOI: http://dx.doi.org/10.1590/S0100-29452013000200014

MUSSURY, R. M.; FERNANDES, W. D.; SCALON, S. P. Q.. Atividade de alguns insetos em flores de Brassica napus em Dourados-MS e a interação com fatores climáticos. Ciência e Agrotecnologia, Lavras, v.27, n.2, p.382-388, 2003. DOI: http://dx.doi.org/10.1590/S1413-70542003000200018

POURIAN, H. R.; HASSANLOUI, R. T.; ASHOURI, A.; LOTFALIZADEH, H. A.; NOZARI, J.. Ontogeny and reproductive biology of Diadegma semiclausum (Hym.: Ichneumonidae), a larval endoparasitoid of Diamondback Moth, Plutella xylostella (Lep.: Plutellidae). Arthropod Structure \& Development, v.44, n.1, p.69-76. 2015. DOI: https://doi.org/10.1016/j.asd.2014.11.002
PRATISSOLI, D.. Diabrotica speciosa. AGROFIT: Sistema de Agrotóxicos Fitossanitários, Ministério da Agricultura, Pecuária e Abastecimento. 2019.

RAFAEL, A. J.; MELO, G. A. R.; CARVALHO, C. J. B.; CASARI, S. A.; CONSTANTINO, R.. Insetos do Brasil: Diversidade Taxonômica. Ribeirão Preto: Holos. 2012

RESENDE, A. L. S.; HARO, M. M.; SILVA, V. F.; SOUZA, B.; SILVEIRA, L. C. P.. Diversidade de predadores em coentro, endro e funcho sob manejo orgânico. Arquivos do Instituto Biológico de São Paulo, Campinas, v.79, n.2, p.193-199, 2012. DOI: http://dx.doi.org/10.1590/S180816572012000200007

RESENDE, A. L. S.; OLIVEIRA, R. J.; LIXA, A. T.; SANTOS, C. M. A.; GUERRA, J. G. M.; MENEZES, E. L. A.. Estrutura populacional de joaninhas predadoras em consorcio couve e coentro em comparação ao monocultivo da couve, sob manejo orgânico. Boletim de Pesquisa e Desenvolvimento. Embrapa Agrobiologia, Seropédica, 2009.

RODRIGUES, H. S.; HOLTZ, A. M; TULER, A. C.; ALVES, D. I.; VIEIRA, L. P.. Broca-da-couve (Hellula phidilealis). In: HOLTZ, A. M.; RONDELLI, V. M.; CELESTINO, F. N.; BESTETE, L. R.; CARVALHO, J. R.. Praga das Brássicas. Colatina: IFES, 2015.

ROOT, R. B.. The niche exploitation pattern of the blue-gray gnatcatcher. Ecological Monographs, v.37, n.4, p.317-350. 1967. DOI: https://doi.org/10.2307/1942327

SATO, M. E.. Manejo e métodos de controle de pragas em folhosas e brássicas. In: HOJO, H.. Programa de Sanidade em Agricultura Familiar. (Boletim Técnico). São Paulo: Instituto Biológico, 2016.

SILVA, V. P.; PEREIRA, M. J. B.; VIVAN, L. M.; MORAES, M. C. B.; LAUMANN, R. A.; BORGES, M.. Monitoramento do percevejo marrom Euschistus heros (Hemiptera: Pentatomidae) por feromônio sexual em lavoura de soja. Pesquisa Agropecuária Brasileira, Brasília, v.49, n.11, p.844852, 2014. DOI: http://doi.org/10.1590/S0100204X2014001100003

SOUZA, L. P.; HOLTZ, A. M.; BOTTI, J. M. C.; FRANZIN, M. L.; PAULO H. H.. Lagarta-rosca (Agrotis ipsilon). In: HOLTZ, A. M.; RONDELLI, V. M.; CELESTINO, F. N.; BESTETE, L. R.; CARVALHO, J. R Praga das Brássicas. Colatina: IFES, 2015.

SUENAGA, H.; HAMAMURA, T.. Occurrence of carabid beetles (Coleoptera: Carabidae) in cabbage fields and their possible impact on lepidopteran pests. Applied Entomology and Zoology, Tokyo, v.36, n.1, p.151-160, 2001. DOI: https://doi.org/10.1303/aez.2001.151

SYED, T. S.; ABRO, G. H.; SHAIKH, M. A.; MAL, B.; SHELTON, A. M.. Parasitism of Plutella xylostella (Lepidoptera: Plutellidae) in southern Pakistan. Florida Entomologist, v.101, n.2, p.172-177, 2018. DOI: https://doi.org/10.1653/024.101.0204.

THULER, R. T.; BORTOLI, S. A.; HOFFMANN-CAMPO, C. B.. Classificação de cultivares de brássicas com relação à resistência à traça-das-crucíferas e à presença de glucosinolatos. Pesquisa Agropecuária Brasileira, Brasília, v.42, n.4, p.467-474, 2007. 
TORRES, C. S. A. S.; TORRES, J. B.; BARROS, R.; PALINI, A.. Parasitismo de traça-das-crucíferas por Oomyzus sokolowskii. Revista Pesquisa Agropecuária Brasileira, Brasília, v.45, n.7, p.638-645, 2010. DOI:

http://dx.doi.org/10.1590/S0100-204X2010000700002

TRINDADE, R. L.; GORAYEB, I. S.. Maruins (Diptera:

Ceratopogonidae: (Culicoides), após a estação chuvosa, na Reserva de Desenvolvimento Sustentável Itatupã-Baquiá,
Gurupá, Pará, Brasil. Revista Pan-Amazônica de Saúde, v.1, n.2, 2010. DOI: http://dx.doi.org/10.5123/S2176$\underline{62232010000200015}$

VAN RIJN, P. C. J.; KOOIJMAN, J.; WÄCKERS, F. L.. The contribution of floral resources and honeydew to the performance of predatory hoverflies (Diptera: Syrphidae). Biological Control, Orlando, v.67, n.1, p.32-38, 2013. https://doi.org/10.1016/j.biocontrol.2013.06.014

A CBPC - Companhia Brasileira de Produção Científica (CNPJ: 11.221.422/0001-03) detém os direitos materiais desta publicação. Os direitos referem-se à publicação do trabalho em qualquer parte do mundo, incluindo os direitos às renovações, expansões e disseminações da contribuiç̃ão, bem como outros direitos subsidiários. Todos os trabalhos publicados eletronicamente poderão posteriormente ser publicados em coletâneas impressas sob coordenação da Sustenere Publishing, da Companhia Brasileira de Produção Científica e seus parceiros autorizados. Os (as) autores (as) preservam os direitos autorais, mas não têm permissão para a publicação da contribuição em outro meio, impresso ou digital, em português ou em tradução. 